\title{
Split-Belt Treadmill Stepping in Infants Suggests Autonomous Pattern Generators for the Left and Right Leg in Humans
}

\author{
Jaynie F. Yang, Erin V. Lamont, and Marco Y. C. Pang \\ Department of Physical Therapy and Centre for Neuroscience, University of Alberta, Edmonton, Alberta, Canada T6G 2G4
}

\begin{abstract}
The behavior of the pattern generator for walking in human infants (7-12 months of age) was studied by supporting the infants to step on a split-belt treadmill. The treadmill belts could be run at the same speed (tied-belt), different speeds, or in different directions (split-belt). We determined whether the legs could operate independently under these conditions, as demonstrated by taking different numbers of steps or by stepping in different directions. Video, surface electromyography, electrogoniometry, and force platform data were recorded. The majority of infants who could step under tied-belt conditions also stepped under split-belt conditions. During forward stepping at low speed differentials between the two belts (ratio, $<4$ ), infants adopted a step cycle duration that was intermediate between that expected from tied-belt stepping at each of the speeds. At large speed differentials between the two belts (ratio, 7-22), the infants took extra steps on the fast leg during the stance phase on the slow leg. When the two belts ran in opposite directions, one leg stepped forward, and the other stepped backward. During all forms of stepping, the legs maintained a reciprocal relationship, so that swing phase occurred in one leg at a time. Timing of muscle activity suggests a strong inhibition between the flexor-generating centers on each side and a weaker inhibition between the extensor-generating centers. The stepping behavior resembled that reported for other animals under similar conditions, suggesting that the pattern generator for each limb is autonomous but interacts with its counterpart for the contralateral limb.
\end{abstract}

Key words: locomotion; central pattern generator; human; development; sensorimotor; movement; motion; motor activity

\section{Introduction}

Pattern generators controlling rhythmic locomotor movements in vertebrates are thought to have a distributed (for review, see Kiehn and Butt, 2003) and modular organization (Grillner and Zangger, 1979; Grillner, 1981; Jordan, 1991; Stein, 2005). Although the constituents of a module are unclear, we know that groups of neurons, such as neurons from each side of the spinal cord (Kudo and Yamada, 1987; Soffe, 1989) or neurons controlling a set of muscles (Cheng et al., 1998; Stein and DanielsMcQueen, 2002, 2004), have considerable rhythmogenic and autonomous ability. Behaviorally, the independence of each hemispinal cord can be reflected in the coordination of leg movements on a split-belt treadmill, in which the belts are run at different speeds. Animals as diverse as cats and stick insects (Forssberg et al., 1980; Foth and Bassler, 1985) maintain coordination while stepping on a split-belt treadmill, but, in addition,

Received May 3, 2005; revised June 15, 2005; accepted June 16, 2005.

This work was supported by a grant from the Canadian Institutes for Health Research (J.F.Y.). E.V.L. was supported in part by an Alberta Government scholarship. We thank R. Vishram for excellent technical support, R. Gramlich and S. Graziano for construction of the split-belt treadmill, and Dr. S. Patrick for technical drawings and figure preparation. We thank Drs. Keir Pearson and Paul Zehr for their helpful suggestions on previous versions of this manuscript.

Correspondence should be addressed to Jaynie F. Yang, Department of Physical Therapy, 2-50 Corbett Hall, University of Alberta, Edmonton, Alberta, Canada T6G 2G4. E-mail: jaynie.yang@ualberta.ca.

DOI:10.1523/JNEUROSCI.1765-05.2005

Copyright $\odot 2005$ Society for Neuroscience $\quad$ 0270-6474/05/256869-08\$15.00/0 their limbs can also operate somewhat independently, as demonstrated by the limb on the faster treadmill belt sometimes taking more steps than the other leg(s) on the slower belt(s) (i.e., asymmetrical stepping). These results indicate that the pattern generators for each leg have some degree of autonomy while interacting with each other for coordination.

There is increasing evidence that the human spinal cord also contains a pattern generator for walking (Calancie et al., 1994; Dimitrijevic et al., 1998). Although it is likely that the human pattern generator for walking has retained some or many of the characteristics found in other vertebrates, it is not a foregone conclusion (Capaday, 2002; Nielsen, 2003). The evolution of bipedal walking has imposed greater demands on the lower limbs, demands that may have been met by evolutionary changes to the pattern generator in the spinal cord, and/or the role of other centers in the nervous system (Capaday et al., 1999; Petersen et al., 2001).

All previous reports of human adults stepping on split-belt treadmills have reported alternate stepping, with left and right steps alternating in a 1:1 manner (Dietz et al., 1994; Prokop et al., 1995; Jensen et al., 1998). No asymmetrical stepping rhythms were reported. However, volitional control may have overridden the behavior of the pattern generator in these adult subjects. In the single study with infants (Thelen et al., 1987), 2:1 steps were mentioned, but they seemed infrequent, and no details were provided. Because the speed differential between the belts was small 
(twofold), the full potential of the pattern generators may not have been revealed.

In this study, we examined split-belt treadmill stepping in infants, because their stepping is less likely to be under strong cortical control compared with adults (Forssberg, 1985; Yang et al., 1998). We tested stepping with a large variation in belt speeds. In addition, we tested the condition in which the belts were run in opposite directions, because other animals show such behavior when making tight turns in walking (Graham, 1985) or swimming (Field and Stein, 1997a,b). Preliminary data have been published (Lamont et al., 2003) and included in a review paper (Yang et al., 2004).

\section{Materials and Methods}

\section{Subjects}

A total of 45 infants, ranging in age from 5.0 to 11.8 months (mean \pm SD; $9.4 \pm 1.3$ months), were studied. None of these infants were able to walk independently at the time of the study. Infants were recruited through local health clinics. Parents were instructed to practice stepping with their infants, as described by Yang et al. (1998). Once the infant was able to perform 10 consecutive steps (as reported by a parent), an experiment was scheduled. Ethical approval for this study was obtained through the Health Research Ethics Board (University of Alberta and Capital Health, Edmonton). Informed and written consent was obtained from a parent before the experiment. All experiments were conducted in accordance with the Declaration of Helsinki for experiments on humans.

\section{Instrumentation}

Walking was studied on a custom-built split-belt treadmill (model INFSBT-FP; designed and built by R. Gramlich and S. Graziano, University of Alberta). The two treadmill belts are driven by separate motors, which could be electrically coupled to run at the same speed (tied-belt condition) or uncoupled to run at different speeds (forward split-belt condition). The belts are also capable of running in the opposite direction (opposite split-belt condition) by reversing the motor drive. The speed of each treadmill belt was estimated by the rotation of the main drive shaft for each belt and was confirmed with the video data. A Plexiglas partition (15 cm in height) was placed between the two belts to ensure that the infant's legs remained on separate belts. Force plates located under each belt recorded vertical ground reaction forces.

Stepping movements were recorded with a video camera (Canon Elura 50; Canon, Mississauga, Ontario, Canada) in the sagittal plane (right side) at 30 frames/s. Infants were dressed in black stockings to enhance the contrast of the white markers $(2 \mathrm{~cm}$ in diameter). Markers were placed over the superior border of the iliac spine, the greater trochanter, the knee joint line, the lateral malleolus and the head of the fifth metatarsal of the right leg, and the medial malleolus and first metatarsal of the left leg. An electrogoniometer (Penny and Giles Biometrics, Blackwood, Gwent, UK) was placed over each hip joint. The arms of the goniometer were aligned with the midaxillary line of the trunk and the longitudinal axis of the femur. The video and analog signals were synchronized by a custom-made timer, which generated pulses at $1 \mathrm{~Hz}$.

In some experiments (17 infants), surface electromyograms (EMGs) were also recorded from the legs bilaterally. Pairs of infant-sized, silversilver chloride EMG electrodes (Kendall LTP, Chicopee, MA) were applied over the tibialis anterior (TA) and gastrocnemius-soleus muscles after the skin was cleaned with alcohol. The signals were conditioned with an AMT-8 amplifier (Bortec Biomedical, Calgary, Alberta, Canada), bandpassed from $10 \mathrm{~Hz}$ to $10 \mathrm{kHz}$. The EMG signals were full-wave rectified and low-pass filtered at $30 \mathrm{~Hz}$ before digitization.

All signals were digitized on-line using a commercially available analog-to-digital program (AxoScope; Molecular Devices, Foster City, CA) at a rate of $340 \mathrm{~Hz}$. The force plate and electrogoniometer signals were also low-pass filtered at $30 \mathrm{~Hz}$. All unprocessed analog signals were also recorded on VHS tape with a pulse code-modulated encoder (Vetter, Rebersburg, PA) for backup.

\section{Experimental Procedures}

The experiments were $\sim 1 \mathrm{~h}$ long. The infant was held under the arms from behind by one of the experimenters or a parent, over the treadmill belts. Forearm supports were provided for the person holding the infant to limit the possibility of imposing movements on the infant. The infant was allowed to support as much of his/her weight as possible. The infant's body weight was measured at the end of the experiment.

Each experiment began with a trial in which both belts ran at the same moderate speed $(0.3-0.6 \mathrm{~m} / \mathrm{s})$ to familiarize the infant with treadmill stepping. The order of subsequent trials (see below) was arbitrarily chosen and typically consisted of one or two of the following.

Forward split-belt stepping. All infants were tested in this condition, with 39 generating useful data. The ratio of the two belt speeds ranged from 1.8 to 22.5 , with the speed of the slow belt ranging between 0.04 and $0.31 \mathrm{~m} / \mathrm{s}$ and the speed of the fast belt ranging between 0.23 and $1.69 \mathrm{~m} / \mathrm{s}$. Not all infants were tested at all speeds. In the first 10 infants tested, we focused on how they adjusted to the belt speeds when they were first put on the treadmill, to determine whether there was a period of learning before a stable pattern was achieved. Only infants who showed at least 15 alternate steps in the first split-belt trial were included.

Matched split-belt and tied-belt speeds in forward stepping. Twelve infants were tested under tied-belt conditions in which the belt speed was matched to the fast and slow belt speeds during the split-belt condition in turn $(<20 \%$ difference between split-belt and tied-belt conditions). In this way, these infants could serve as their own control for comparisons of tied- and split-belt stepping. In four other infants, we occasionally stopped one leg on the split-belt treadmill by placing a piece of cardboard under the foot and holding it stationary [Pang and Yang (2000), their Fig. 2]. We anticipated the stepping rate in the contralateral leg to change back to what would be expected under tied-belt conditions when the influence of the opposite leg was removed.

Treadmill belts running in opposite directions. Ten infants were studied with the right treadmill belt running backward, while the left was running forward. The speeds of the two belts were the same under this condition and ranged between 0.25 and $0.4 \mathrm{~m} / \mathrm{s}$.

\section{Data analysis}

Successful sequences of forward stepping (at least three consecutive steps) were identified off-line from video. Opposite direction stepping was deemed successful only if the backward-stepping foot was placed behind the hip joint marker to initiate stance phase (Lamb and Yang, 2000) and if a minimum of three steps were seen on each side in the sequence.

The onset of stance was defined as the time when the foot made contact with the treadmill. The onset of swing was defined as the time when the toe marker changed from moving backward (stance phase) to moving forward (swing phase) for forward stepping or visa versa for backward stepping. Force plate data provided confirmation of the onset of stance and swing phases. If there were discrepancies between the video and force plate data, the video data were used. Individual steps were selected from the digitized data and analyzed using custom-written programs in Matlab (MathWorks, Natick, MA). Signals from the selected steps were averaged, and stance and swing durations for each step were calculated, as were the average level of force from the force plates during the stance phase and the average level of muscle activity during the stance and swing phases.

The number of steps using either alternate or asymmetrical coordination was determined and expressed as a percentage of the total steps. Step counts always referred to steps made by the leg on the slow belt. In addition, a phase lag was calculated by expressing the onset of the step cycle in one leg as a function of the step cycle of the contralateral leg, and expressed as a percentage. When there were more steps on the fast belt, those steps were expressed as a function of the leg on the slower belt. Although phase lag is technically an inappropriate term for asymmetrical steps, because the frequency of stepping is different on each side, we will still use this term to refer to the coupling for simplicity.

Asymmetrically coupled steps are particularly suited for studying the relationship between the flexor and extensor-generating centers for each leg. Only subjects with a sequence of asymmetrical steps (more than two 
in a row) and EMG signals free of crosstalk were used for this analysis. Crosstalk was defined as a Pearson's product-moment correlation $>0.2$ between the flexor and extensor muscles on a leg in the sequence of interest. EMG onset and offset times were estimated visually based on crossing a threshold that defined noise level. The duration of coactivity between homologous muscles from the two legs was expressed as a percentage of the total sequence time.

\section{Statistics}

To determine whether there were time-dependent effects (i.e., learning) when subjects were first exposed to split-belt stepping, average step cycle durations were calculated for groups of three steps each, in chronological order. The first three groups of steps and the last two groups were compared with a repeated-measures ANOVA. Paired $t$ tests were used for post $h o c$ analysis. Paired $t$ tests were also used for the comparison of step cycle, stance, and swing phase durations under different experimental conditions in which the subject served as his own control. Comparison of the cycle structure in the fast leg for asymmetrically coupled steps were made with a paired $t$ test, and a repeated-measures ANOVA for the 2:1 and 3:1 coupled steps, respectively. An independent $t$ test was used to compare coactivation levels between homologous muscles on the two sides during asymmetrically coupled steps. The level of significance was 0.05 for all comparisons, except for the post hoc tests, which were adjusted for the number of comparisons.

\section{Results}

\section{Success rate of split-belt walking}

The majority of infants (39 of 45 infants) who could step on the treadmill with both belts running at the same speed could also step when the belts were running at different speeds. The six infants who were unsuccessful still showed some stepping, but the sequences of stepping were not long enough to meet our definition of a successful trial. When the two belts were running in opposite directions, 6 of 10 of the infants who could step in the tied-belt condition were also successful in this task. The four infants who were not successful also showed some stepping with the belts running in opposite directions, but, in two infants, the sequences were too short to be included in the analysis, and the other two failed to meet our definition of backward stepping. In summary, the ability to step under split-belt conditions was very high.

\section{Time-dependent adaptation}

Infants adjusted rapidly to the split-belt condition, which ranged from a speed differential of 2-13 (average, 5.2). There was a trend for the first three step cycles to be slightly shorter in duration than the others. The one-way repeated-measures ANOVA on cycle duration was significant for the slow leg only. Among the many post hoc comparisons, only the average of the first three steps on the slower treadmill belt was significantly different from the other groups of steps. Thus, aside from the first three steps, all steps were representative of the steady state split-belt stepping.

\section{Forward split-belt walking}

Infants could adapt to large differentials between the two belt speeds. The maximum speed ratio between the two belts accommodated was 22.5. When the speed differential of the belts was low, infants tended to maintain alternate stepping by changing their cycle structure and duration to accommodate the differences (see below). As the differential increased, the infants sometimes used asymmetrical steps (i.e., 2:1, 3:1, or other). The coupling pattern varied step to step, with alternate steps randomly mixed with asymmetrical steps. A typical example is shown in Figure 1. The force plate data show when the legs were in stance and swing phase. The activity of a flexor and extensor muscle is
Subject Code: OZR $(8.5 \mathrm{~m})$

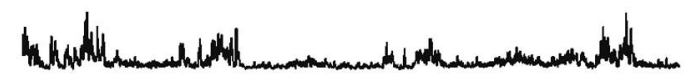

RTA

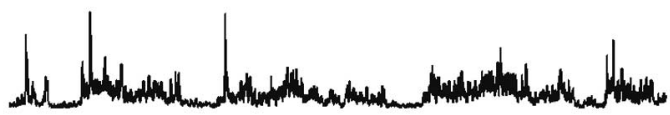

RSOL
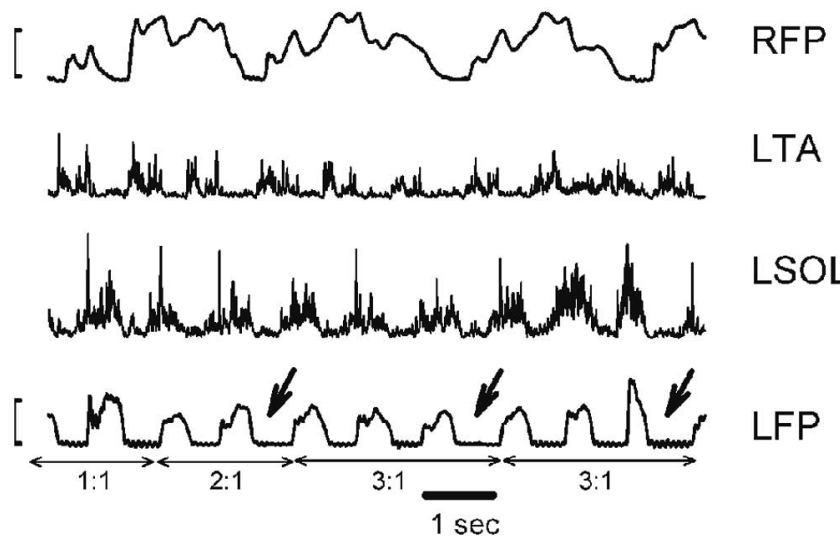

Figure 1. Typical data from a single subject ( 8.5 months of age) during split-belt treadmill walking. The belt speeds were 0.06 and $0.49 \mathrm{~m} / \mathrm{s}$ for the right $(\mathrm{R})$ and left $(\mathrm{L})$ sides, respectively. Gastroc-soleus (SOL) and TA EMGs are shown together with force platform (FP) data. In this sequence of walking, the subject showed a mixture of 1:1, 2:1, and 3:1 coordination between the legs. The EMGs are in arbitrary units, and the vertical brackets to the left of the force plate traces indicate $50 \%$ body weight. Diagonal arrows point to the second and third steps of the leg on the fast belt during 2:1 and 3:1 steps. These steps were systematically longer than the others.

shown for each leg. The coupling pattern is shown at the bottom of the graph. In general, the occurrence of asymmetrically coupled steps was more common with larger speed differentials between the two belts (Fig. 2). Steps with 2:1 and 3:1 patterns were common, and 4:1 and 5:1 steps were occasionally observed. The ratio of fast-to-slow steps was always an integer.

\section{One-to-one coupling}

During alternate stepping on the split-belt treadmill, the step cycle duration was intermediate between that during tied-belt stepping at each of the belt speeds. Figure $3 A$ illustrates the step cycle duration as a function of the treadmill belt speed for all of the trials recorded. Tied-belt stepping is indicated by the black circles. The data from the tied-belt condition was well fit by a power curve $\left(y=a x^{-b}\right)$, as shown previously (Yang et al., 1998). In Figure 3, split-belt stepping is indicated by the lighter symbols, with the step cycles from the slow belt indicated by gray symbols, and those from the fast belt indicated by white symbols. Each symbol represents an average from a specific stepping condition in an infant. Most infants provided a number of data points, because stepping was obtained under a few conditions. It is clear that the adaptation to split-belt walking was made by both legs, with the leg on the fast belt taking a slower step than it normally would at that speed under tied-belt conditions (Fig. 3A, many white circles above black circles) while the leg on the slow belt did the opposite (many gray circles below the black circles). The leg on the fast belt slows down by lengthening its swing phase (Fig. $3 B$, many white circles above black circles), while the leg on the slow belt speeds up by shortening the stance phase (Fig. 3C, many gray circles below black circles). Generally, the changes were more dramatic in the leg on the slower treadmill belt, such that the average step cycle duration was closer to what would be ex- 


\section{Interlimb Coupling Pattern}

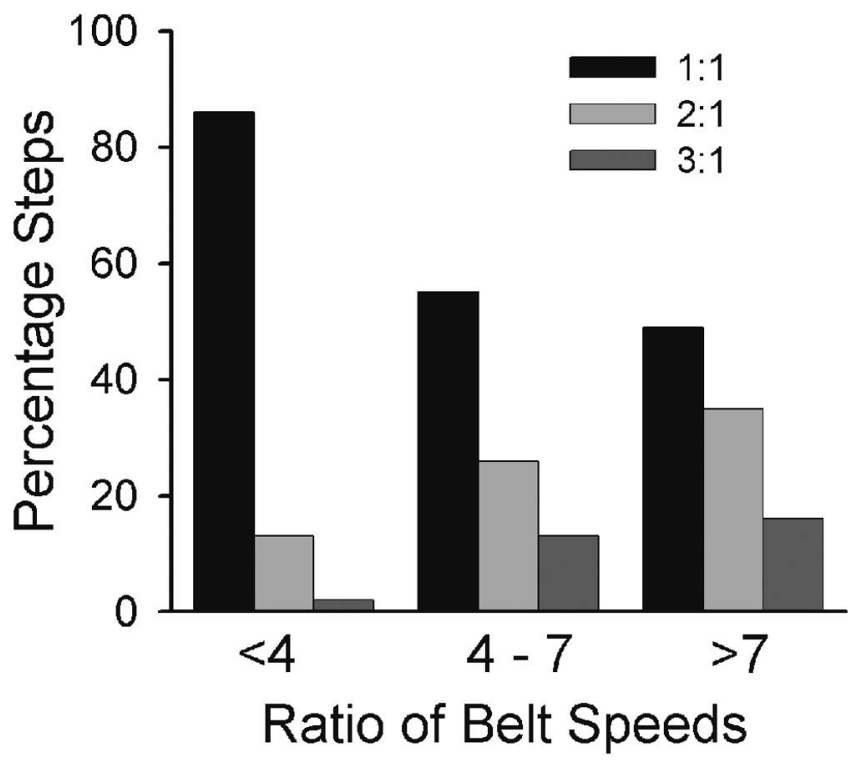

Figure 2. Ratio of treadmill belt speed influences the type of interlimb coordination. The percentage of steps showing 2:1 or 3:1 coordination increases as the ratio of belt speed increases. All infants included in this figure provided data with belt speed ratio in the range of $<4$ and at least one trial with $>4$ ( $n=24$ infants).
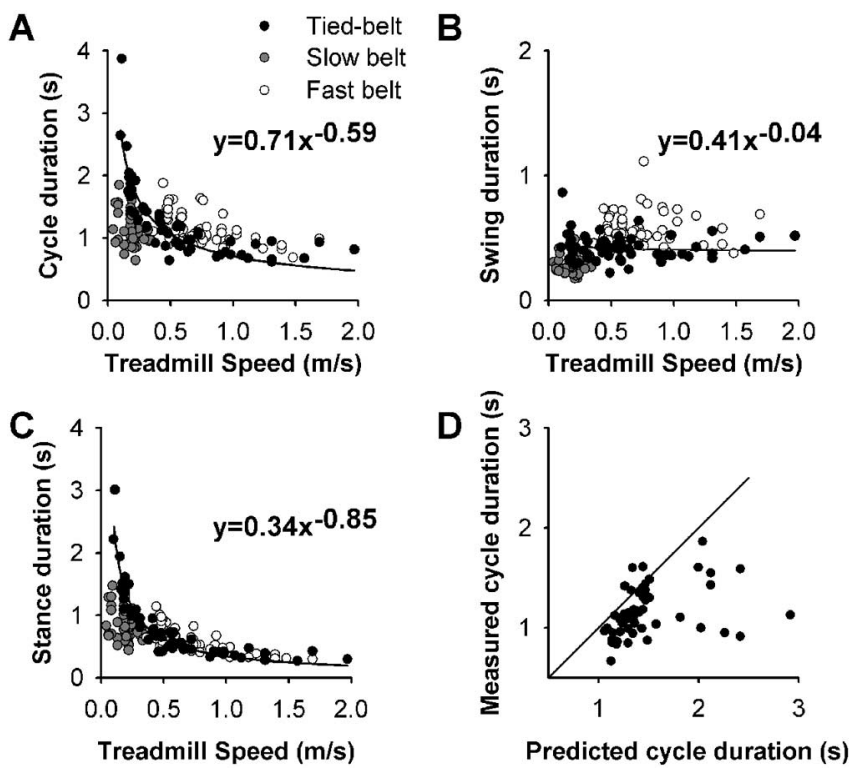

Figure 3. Step cycles during tied and split-belt stepping. $\boldsymbol{A}-\boldsymbol{C}$, The step cycle $(\boldsymbol{A})$, swing $(\boldsymbol{B})$, and stance $(\boldsymbol{C})$ phase durations are shown as a function of treadmill speed. Tied-belt measures (black circles) were fitted with the power function $y=a x^{-b}$, shown as the solid line on the graph, with parameters for the best-fitting line displayed. The measures taken from split-belt stepping are indicated by gray circles for the slow leg and white circles for the fast leg $(n=30$ infants). Each point represents an average of at least eight steps in a trial. $\boldsymbol{D}$, Comparison of the measured and predicted cycle duration. The predicted cycle duration is the mathematical average of that estimated from measures obtained during tied-belt stepping, using the equation in $\boldsymbol{A}$. The unit line indicating perfect prediction is shown. Most measured durations were shorter than the predicted.

pected on the fast belt. This is seen by plotting the average step cycle duration measured during split-belt stepping against the predicted cycle duration (Fig. 3D). The predicted cycle duration is the mathematical average of the cycle duration during tied-belt
A $\quad$ B

B C ए Tied
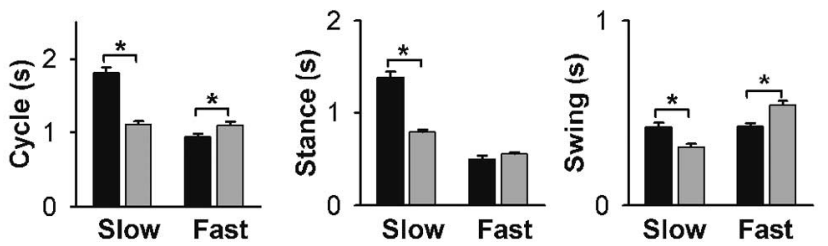

Figure 4. Cycle structure in tied and split-belt stepping with matched treadmill speeds. The average step cycle, stance, and swing phase durations for infants $(n=12)$ with exactly matched speeds in tied-belt and split-belt conditions are shown for steps exhibiting 1:1 coordination (means \pm SEM). Black bars were measured from two separate trials during tied-belt stepping (i.e., fast and slow speeds). Gray bars were measured from split-belt stepping with one belt at the fast and one at the slow speed. $A$, The step cycle duration during tied-belt stepping is very different for the fast compared with the slow speed (i.e., height of black bars). When stepping under split-belt conditions, the step cycle on the two sides become similar (i.e., height of gray bars). The cycle duration changes more for the leg on the slow belt compared with that on the fast belt during split-belt stepping (difference greater between black and gray bars on the left compared with the right). $A-C$, During split-belt stepping, shortening of the step cycle on the slow belt $(\boldsymbol{A}$, left) comes from shortening of the stance phase (B, left) and the swing phase ( $\boldsymbol{C}$, left). The slight lengthening of the step cycle in the leg on the fast belt ( $\boldsymbol{A}$, right) comes mostly from lengthening of the swing phase $(\boldsymbol{C}$, right). The asterisk indicates statistical significance (paired $t$ tests). Error bars represent SEM.

stepping at each of the treadmill speeds, estimated from the equation $y=0.71 x^{-0.59}$ in Figure $3 A$. If the prediction is correct, the points should fall on or near the unity line. The vast majority of points fell below the line, indicating that the measured cycle durations were shorter than the predicted.

Comparison of the cycle, stance, and swing phase durations under the tied- and split-belt conditions with matched speeds are shown in Figure 4. Measures obtained from the leg on the slow belt and the fast belt are shown. Note that tied-belt measures were obtained from two separate trials, whereas split-belt measures were obtained from the same trial. It is clear that the step cycle duration during split-belt stepping is much closer to that during tied-belt stepping at the fast speed (Fig. $4 \mathrm{~A}$ ), indicating a greater adjustment made by the leg on the slow belt. This adjustment included shortening of both the stance phase (Fig. $4 \mathrm{~B}$, left) and swing phase (Fig. 4C, left) during split-belt stepping. The leg on the fast belt lengthened its cycle duration (Fig. $4 A$, right) mostly by lengthening the swing phase duration (Fig. $4 C$, right). To verify the influence of the pattern generator of one leg affecting that of the other on a moment-by-moment basis, we occasionally stopped the stepping of one leg during forward split-belt stepping. Under this situation, the step cycle on the contralateral side reverted immediately back to what was observed during tied-belt stepping at the same speed (data not shown).

\section{Asymmetric coupling}

The infants performed asymmetric stepping by increasing the step cycle duration on the slow side while the fast side continued stepping at much the same rate as that during 1:1 coupling. This was easily seen from the raw data (Fig. 1). The cycle duration is lengthened on the slow side primarily by lengthening the stance phase, such that the stance phase occupied a disproportionately long period of the step cycle. Paired $t$ tests showed significant lengthening of the stance phase across subjects and no change in the swing phase (data not shown).

The multiple step cycles on the fast side were not equal in length (Fig. 5A), with the second fast step in 2:1 coupling and the third fast step in 3:1 coupling being longer. The duration of the stance phase in that step was very slightly lengthened, and it cor- 
A
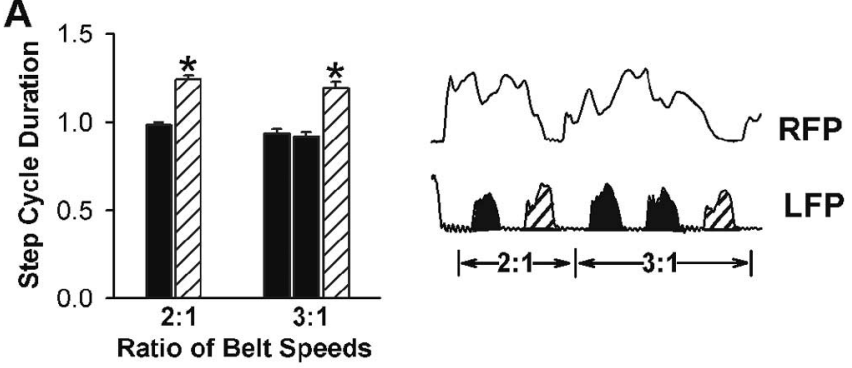

Ratio of Belt Speed 2:1
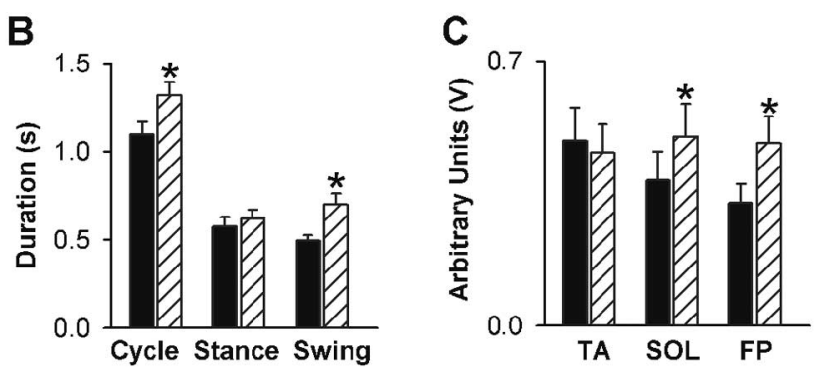

Figure 5. Cycle structure and muscle activation in asymmetrically coupled steps. $\boldsymbol{A}$, In asymmetrically coupled steps, the step cycles on the fast belt were not identical in duration. An example of raw data from the left force plate (LFP) and right force plate (RFP) are shown on the right, to illustrate the shading of the different steps. The step that corresponded to swing phase on the contralateral side (hatched bars) was significantly longer than the other steps (filled bars). The bar graph shows mean \pm SEM with statistically significant differences (asterisk) ( $n=250$ steps from 33 infants for 2:1 steps, and $n=90$ steps from 27 infants for 3:1 steps). $\boldsymbol{B}$, A subset of the data from $\boldsymbol{A}$, which consisted of 2:1 steps that were followed by a 2:1 or 3:1 step are shown. The difference in cycle duration for the first and second step in the fast leg remained. This difference was essentially a result of a longer swing phase. $\boldsymbol{C}$, The average gastroc-soleus (SOL) EMG amplitude and the average weight bearing was higher in the stance phase of the second step, whereas the average TA EMG was not different between the two steps. FP, Force plate. Error bars represent SEM.

responded in time to the contralateral swing phase. The subsequent swing phase was also longer (Fig. 1, arrows in single subject data). To ensure that this difference (particularly the length of the swing phase) was not a result of the stepping condition in the subsequent step (which could be a 1:1 coupled step), we reanalyzed a subset of the 2:1 steps using only those steps that were followed by another $2: 1$ or 3:1 coupled step. The 3:1 steps were not analyzed this way because of insufficient numbers. With this analysis, the differences remained (Fig. 5B). We determined whether the difference in phase durations was accompanied by differences in the intensity of muscle activation or the amount of weight bearing. The average muscle activity for the tibialis anterior (swing phase) and gastroc-soleus (stance phase), and the average force plate signal (stance phase) were calculated for each of the fast steps during 2:1 coupling. There were no significant differences in the TA activation between the two fast steps in the swing phase, but there were significant differences in both the amount of weight bearing (force plate signal) and the gastrocsoleus EMG during the stance phase (Fig. 5C).

The extent to which homologous muscles from the legs were coactive was estimated. Example from individual sequences are shown in Figure 1. There was significantly more coactivation of extensors (mean $\pm \mathrm{SD} ; 31 \pm 2 \%$ ) compared with flexors $(13 \pm$ $4 \%$ ) (Student's $t$ test).

Regardless of the type of coupling between the legs, the stepping was very coordinated. For the most part, one leg went into
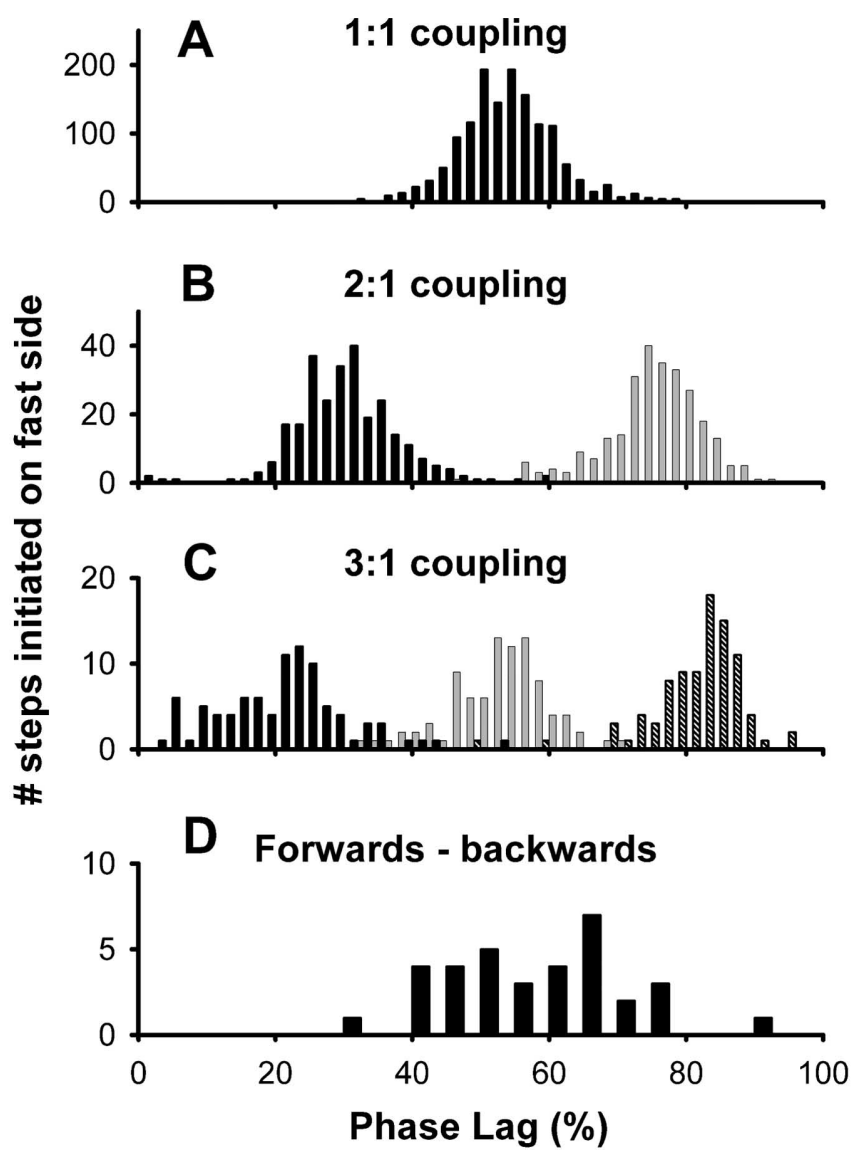

Figure 6. Phase relationship between the two legs for different types of stepping. Initiation of steps on the fast leg as a function of the step cycle on the slow leg is defined as the phase lag. A phase lag of $0 \%$ means the two steps were initiated simultaneously, and a phase lag of $50 \%$ means the steps were exactly alternate. $\boldsymbol{A}$, Steps that were coupled 1:1 show a phase lag centered $\sim 50 \%$. Phase lags for steps coupled 2:1 and 3:1 are shown in $\boldsymbol{B}$ and $\boldsymbol{C}$, respectively, with each step on the fast side represented by different shading. Data obtained from 1416 steps with 1:1 coupling from 35 infants, 269 steps with 2:1 coupling from 32 infants, and 90 steps with 3:1 coupling from 27 infants. $\boldsymbol{D}$ shows coupling when the two belts were running in opposite directions. Phase lag here refers to the initiation of the forward-stepping leg as a function of the backward stepping leg. Data are from 31 steps in six infants.

swing phase only when the contralateral leg was in the stance phase. Figure $6 A$ shows that when the infants were stepping with an alternating pattern (1:1 coupling), regardless of the speed differential, the phase lag was close to $50 \%$ (mean, 53\%). The phase lag was further analyzed for different split-belt speed ratios to determine whether the phase interval was a function of the speed differential. No relationship was found. During asymmetrically coupled steps, the phase lags for each fast step is shown in different shading (Fig. $6 B, C$ ). There was considerable range in the phase lag for each of the steps, with means of 30 and $74 \%$ for $2: 1$ coupling and 22,52 , and $82 \%$ for $3: 1$ coupling. There were relatively few steps with a phase lag near 0 and $100 \%$, which is the time the contralateral leg on the slow belt entered the swing phase.

\section{Stepping in opposite directions}

Stepping in opposite directions is depicted in Figure 7 for a single subject. The force plate and goniometer signals are shown at the top left. To illustrate the coordination more clearly, five specific time points (A-E) are identified with dotted lines, and the corresponding position and motion of the legs are shown in the 
sketches, made from the corresponding video frames. The position of the heel marker is also illustrated for the frames from $\mathrm{A}$ to $\mathrm{E}$ in the top right of Figure 7, with arrows indicating the direction of motion. While stepping in this manner, infants maintained an alternate relationship between the limbs. The phase lag is shown for all subjects in Figure $6 D$. The forward-stepping side initiated stance phase approximately midway through the step cycle on the backward-stepping side (mean phase interval, 54\%). Thus, the reciprocal relationship between the legs seen during other forms of stepping was also seen when the legs stepped in opposite directions.

\section{Discussion}

We showed for the first time that infants can step in a coordinated manner on a split-belt treadmill with the belts running at remarkably different speeds (up to 22fold difference) and when the belts are running in opposite directions. The two legs could adopt different patterns of stepping, but they remained coordinated so that only one leg entered the swing phase at a time.

\section{Interdependence of the pattern generators for each limb}

The stepping in each limb showed some dependence on its counterpart on the other side. When the two belt speeds were different, a step cycle duration was adopted by each leg that was intermediate between that seen during tied-belt stepping at each of the belt speeds, just as that shown for a variety of other animals (Kulagin and Shik, 1970; Forssberg et al., 1980; Halbertsma, 1983; Foth and Bassler, 1985). Moreover, if one limb was temporarily held stationary in the stance phase during split-belt stepping, the other limb immediately returned to its preferred cycle duration seen during tied-belt stepping. Thus, the influence of one limb on the other is clear, reminiscent of von Holst's (1973) demonstration of "coupled oscillators" controlling fish fins.

Another example of the interdependence of the two limbs is seen during asymmetrically coupled steps. During 2:1 and 3:1 steps, the step cycles of the fast leg were not identical. The stance phase on the fast side coinciding with swing phase on the contralateral side was slightly longer than the others. Moreover, the subsequent swing phase on the fast leg, which corresponded to the beginning of the stance phase on the slow leg, was also substantially longer than the other swing phases. A similar asymmetry has been reported for split-belt stepping in intact cats (Halbertsma, 1983) and can be seen in the raw data reported for chronic spinal cats [Forssberg et al. (1980), their Figs. 7, 8]. Such interaction between rhythm generators on each side of the body has also been reported for fictive locomotion in acute spinal cats (Grillner and Zangger, 1979) and fictive scratching in spinal turtles (Stein and McCullough, 1998). Lengthening of that step could be explained by either central or peripheral factors. Peripheral factors that might lengthen the stance phase include the higher load borne in that step because the contralateral limb is in swing phase, compared with other steps when the contralateral

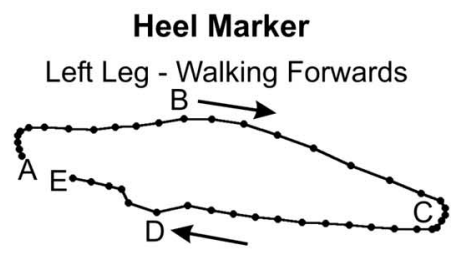

Right Leg - Walking Backwards
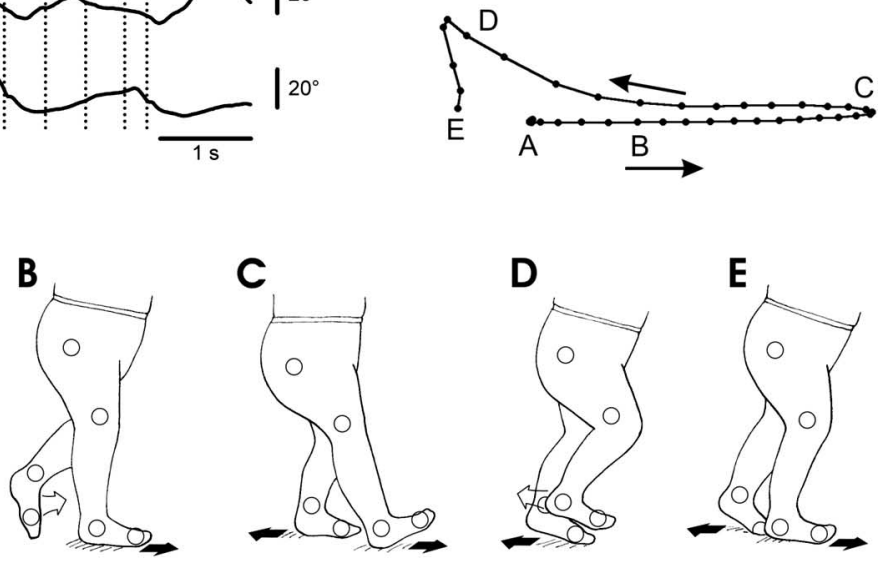

Figure 7. Data from a single subject during opposite direction split-belt stepping. The left leg was stepping forward while the right leg was stepping backward. Sketches from the video frames corresponding to five specific time points (A-E) during the motion of foot during stance phase, and open arrows show motion of foot during swing phase). The corresponding marker in the sagittal plane is shown in the top right for the time interval from times $A-E$, with arrows indicating the direction of flex, flexion; ext, extension.

limb is in the stance phase. Peripheral factors that might lengthen the subsequent swing phase are less clear. Lengthening of the swing phase is a very strong effect, and is not accompanied by stronger activation of the TA muscle. It is interesting that a similar lengthening of the flexor burst duration is seen in fictive preparations (Stein and McCullough, 1998), suggesting central factors may also play a role.

The relationship between the pattern generators on each side is also reflected in the degree to which homologous muscles on each leg could be coactive during stepping. This is most clearly seen during asymmetrically coupled steps when the pattern generators are not operating in a strictly reciprocal way. Based on the timing of muscle activity, we found a much greater degree of coactivity in the extensors on the two sides compared with the flexors. In many sets of raw data, however, there was some depression in the extensor EMG when the contralateral extensor is active (some evidence is shown in Fig. 1). This phenomenon could be explained by changes in sensory input, because the limb load is also varying in exactly the same way (see force plate signal). Overall, there seems to be a stronger reciprocal inhibition between the flexor-generating centers on each side compared with the extensor-generating centers, just as that shown in cats (Forssberg et al., 1980; Hiebert et al., 1996).

\section{Independence of the pattern generator for each limb}

The pattern generators for each limb were also able to operate independently, because at more extreme differences in belt speed, the fast limb could take more steps than the slow limb. This type of flexible coordination has been reported for many animal preparations, including stick insects (Foth and Bassler, 1985), spinal (Forssberg et al., 1980), decerebrate (Kulagin and Shik, 1970) and 
intact cats (Halbertsma, 1983), and spinal turtles (Stein and McCullough, 1998). Split-belt studies in human adults showed some independence of the pattern generators for each leg in relation to learning (Prokop et al., 1995), but asymmetrical stepping has not been reported. We confirm here that the putative human infant pattern generator for each limb shows some autonomy much like that in other animals. We attribute the scarcity of asymmetrical stepping in previous split-belt studies in humans to the lower speed differentials used (Thelen et al., 1987; Dietz et al., 1994) and to intervention from higher centers in the nervous system in the case of adults, perhaps to maintain equilibrium. It remains to be seen whether the adult pattern generator retains such independence as seen here with infants.

We also showed that the two limbs can step in opposite directions simultaneously. The stepping is not passive, because we defined successful steps to be those in which placement of the stepping limb at the time of foot-ground contact to be in front of (for forward stepping) or behind (for backward stepping) the hip joint, which requires an active motion. Additional evidence that this is an active process is the fact that swing phase does not coexist on the two sides during this stepping, just like the other forms of stepping. This type of locomotion, in which the legs on each side of the body operate in opposite directions, has been reported for insects pivoting or making very tight turns (for review, see Graham, 1985) and for spinal turtles making turns during swimming (Field and Stein, 1997a,b). Adult humans rarely show this type of coordination, but the capability is present. Perhaps this is a reflection of our evolutionary history.

The ability to step forward on one limb and backward in the other implies that the relative weighting of sensory input to the pattern generator is highly flexible. For example, one of the important sensory signals to initiate swing phase is hip extension for the forward-stepping leg and a hip flexion for the backwardstepping leg (Pang and Yang, 2002). Hence, swing phase is initiated by opposite sensory signals from the hip for each leg. This is similar to walking sideways, when the motion of the two limbs are opposite, with swing phase triggered by hip adduction in the leading limb and hip abduction in the trailing limb (Pang and Yang, 2002). Together, these data suggest that the pattern generator might be better thought of as composing a swing phase controller and a stance phase controller rather than the more traditional flexor and extensor half-center. The swing phase controller can be functionally coupled to whatever muscles are needed to generate the required movement, such as hip flexors, extensors, abductors, or adductors. Moreover, each of the controllers can be inhibited or excited by the appropriate sensory input for the particular walking direction. We speculate that the pattern generators have considerable flexibility in which muscles they control, how they relate to each other, and how sensory input affects them.

\section{Adjustments in step cycle to accommodate split-belt stepping}

During alternate 1:1 stepping on the split-belt treadmill, the intermediate value of the step duration was almost always closer to that in tied-belt stepping at the faster speed. This suggests the faster leg exerted a greater influence on the overall pattern of walking, just as in adults (Dietz et al., 1994). Perhaps the sensory input from the fast leg is stronger and therefore more dominant.

In infants, adjustments in the step cycle occurred in both stance and swing phases in split-belt stepping. During 1:1 stepping, for example, the stance phase duration was longer in the slow leg, while the swing phase duration was longer in the fast leg (Fig. 4). Hence, the proportion of the step cycle spent in the stance and swing phases are changeable, just as that reported by Forssberg et al. (1980). Our data do not support the idea that the step cycle structure is immutable (Kulagin and Shik, 1970). The stance phase, however, appears to be more flexible in duration than the swing phase.

\section{Conclusions}

The pattern generators for human infant stepping behave in a remarkably similar way to that in other terrestrial animals. The pattern generator for each leg has some autonomy, because different types of coupling (such as 2:1 and 3:1) and opposite directions of stepping are possible simultaneously in both legs. At the same time, the pattern generator for each leg communicates with the other to ensure that swing phase occurs in one limb at a time. The flexor-generating centers in each leg likely inhibit each other, because coexistence of flexor activity is low. In contrast, coexistence of extensor activity on both sides was relatively common. Because different types of coupling are possible between the legs and even opposite directions of walking, it suggests that the coupling relationship among the pattern generators is extremely flexible.

\section{References}

Calancie B, Needham-Shropshire B, Jacobs P, Willer K, Zych G, Green BA (1994) Involuntary stepping after chronic spinal cord injury. Evidence for a central rhythm generator for locomotion in man. Brain 117:1143-1159.

Capaday C (2002) The special nature of human walking and its neural control. Trends Neurosci 25:370-376.

Capaday C, Lavoie BA, Barbeau H, Schneider C, Bonnard M (1999) Studies on the corticospinal control of human walking. I. Responses to focal transcranial magnetic stimulation of the motor cortex. J Neurophysiol 81:129-139.

Cheng J, Stein RB, Jovanovic K, Yoshida K, Bennett DJ, Han Y (1998) Identification, localization, and modulation of neural networks for walking in the mudpuppy (Necturus maculatus) spinal cord. J Neurosci 18:4295-4304.

Dietz V, Zijlstra W, Duysens J (1994) Human neuronal interlimb coordination during split-belt locomotion. Exp Brain Res 101:513-520.

Dimitrijevic MR, Gerasimenko Y, Pinter MM (1998) Evidence for a spinal central pattern generator in humans. Ann NY Acad Sci 860:360-376.

Field EC, Stein PS (1997a) Spinal cord coordination of hindlimb movements in the turtle: intralimb temporal relationships during scratching and swimming. J Neurophysiol 78:1394-1403.

Field EC, Stein PS (1997b) Spinal cord coordination of hindlimb movements in the turtle: interlimb temporal relationships during bilateral scratching and swimming. J Neurophysiol 78:1404-1413.

Forssberg H (1985) Ontogeny of human locomotor control. I. Infant stepping, supported locomotion and transition to independent locomotion. Exp Brain Res 57:480-493.

Forssberg H, Grillner S, Halbertsma J, Rossignol S (1980) The locomotion of the low spinal cat. II. Interlimb coordination. Acta Physiol Scand 108:283-295.

Foth E, Bassler U (1985) Leg movements of stick insects walking with five legs on a treadwheel and with one leg on a motor driven belt. II. Leg coordination when step-frequencies differ from leg to leg. Biol Cybern 51:319-324.

Graham D (1985) Pattern and control of walking in insects. Adv Insect Physiol 18:31-140.

Grillner S (1981) Control of locomotion in bipeds, tetrapods and fish. In: Handbook of physiology-the nervous system II (Brooks V, ed), pp 1179-1236. Baltimore, MD: Waverly.

Grillner S, Zangger P (1979) On the central generation of locomotion in the low spinal cat. Exp Brain Res 34:241-261.

Halbertsma JM (1983) The stride cycle of the cat: the modelling of locomotion by computerized analysis of automatic recordings. Acta Physiol Scand Suppl 521:1-75.

Hiebert GW, Whelan PJ, Prochazka A, Pearson PG (1996) Contribution of hind limb flexor muscle afferents to the timing of phase transitions in the cat step cycle. J Neurophysiol 75:1126-1137. 
Jensen L, Prokop T, Dietz V (1998) Adaptational effects during human split-belt walking: influence of afferent input. Exp Brain Res 118:126-130.

Jordan LM (1991) Brainstem and spinal cord mechanisms for the initiation of locomotion. In: Neurobiological basis of human locomotion (Shimamura M, Grillner S, Edgerton VR, eds), pp 3-20. Tokyo: Japan Scientific Societies.

Kiehn O, Butt SJB (2003) Physiological, anatomical and genetic identification of CPG neurons in the developing mammalian spinal cord. Prog Neurobiol 70:347-361.

Kudo N, Yamada T (1987) Morphological and physiological studies of development of the monosynaptic reflex pathway in the rat lumbar spinal cord. J Physiol (Lond) 389:441-459.

Kulagin AS, Shik ML (1970) Interaction of symmetrical limbs during controlled locomotion. Biofizika 15:164-170.

Lamb T, Yang JF (2000) Could different directions of infant stepping be controlled by the same locomotor central pattern generator? J Neurophysiol 83:2814-2824.

Lamont E, Pang MYC, Yang JF (2003) Adaptation to split-belt walking in human infants. Soc Neurosci Abstr 29:824.4.

Nielsen JB (2003) How we walk: central control of muscle activity during human walking. The Neuroscientist 9:195-204.

Pang MYC, Yang JF (2000) The initiation of the swing phase in human infant stepping: importance of hip position and leg loading. J Physiol (Lond) 528:389-404.

Pang MYC, Yang JF (2002) Sensory gating for the initiation of the swing phase in different directions of human infant stepping. J Neurosci 22:5734-5740.

Petersen NT, Butler JE, Marchand-Pauvert V, Fisher R, Ledebt A, Pyndt HS,
Hansen NL, Nielsen JB (2001) Suppression of EMG activity by transcranial magnetic stimulation in human subjects during walking. J Physiol (Lond) 537:651-656.

Prokop T, Berger W, Zijlstra W, Dietz V (1995) Adaptational and learning processes during human split-belt locomotion: interaction between central mechanisms and afferent input. Exp Brain Res 106:449-456.

Soffe SR (1989) Roles of glycinergic inhibition and $N$-methyl-D-aspartate receptor mediated excitation in the locomotor rhythmicity of one half of the Xenopus embryo central nervous system. Eur J Neurosci 1:561-571.

Stein PS (2005) Neuronal control of turtle hindlimb motor rhythms. J Comp Physiol A Neuroethol Sens Neural Behav Physiol 191:213-229.

Stein PS, Daniels-McQueen S (2002) Modular organization of turtle spinal interneurons during normal and deletion fictive rostral scratching. J Neurosci 22:6800-6809.

Stein PS, Daniels-McQueen (2004) Variations in motor patterns during fictive rostral scratching in the turtle: knee-related deletions. J Neurophysiol 91:2380-2384.

Stein PS, McCullough ML (1998) Example of 2:1 interlimb coordination during fictive rostral scratching in a spinal turtle. J Neurophysiol 79:1132-1134.

Thelen E, Ulrich BD, Niles D (1987) Bilateral coordination in human infants: stepping on a split-belt treadmill. J Exp Psychol 13:405-410.

von Holst E (1973) The behavioural physiology of animals and man, Vol 1, Chap 2. London: Butler and Tanner.

Yang JF, Stephens MJ, Vishram R (1998) Infant stepping: a method to study the sensory control of human walking. J Physiol (Lond) 507:927-937.

Yang JF, Lam T, Pang MYC, Lamont E, Musselman K, Seinen E (2004) Infant stepping: a window to the behaviour of the human pattern generator for walking. Can J Physiol Pharmacol 82:662-674. 